\title{
Change Detection in Optical Aerial Images by a Multi-Layer Conditional Mixed Markov Model
}

Csaba Benedek ${ }^{12} \quad$ Tamás Szirányi $^{1}$

${ }^{1}$ Distributed Events Analysis Research Group Computer and Automation Research Institute, Hungary

${ }^{2}$ ARIANA joint Project-team INRIA/CNRS/UNSA, Sophia Antipolis, France

OMTA SZTAKI RINRIA

Seminar at Florida State University, Department of Statistics, Tallahassee, 16 December 2008 


\section{Content}

(1) Introduction

(2) Feature extraction and integration

- Global intensity statistics

- Local block correlation

- Feature integration

(3) A Mixed Markovian image segmentation model

- Introduction to mixed Markov models

- Proposed model

(4) Experiments 


\section{Introduction}

\section{Content}

\section{(1) Introduction}

(2) Feature extraction and integration

- Global intensity statistics

- Local block correlation

- Feature integration

A Mixed Markovian image segmentation model

- Introduction to mixed Markov models

- Proposed model

Experiments 


\section{Introduction, research goals}

- Change detection in optical aerial image pairs

- new built-up regions, building operations

- planting of trees, fresh plough-land

- groundwork before building-over etc

- Large (many years) time differences $\rightarrow$ different seasons, illumination conditions, vegetations etc.

- Input - preliminary registered orthophotos:

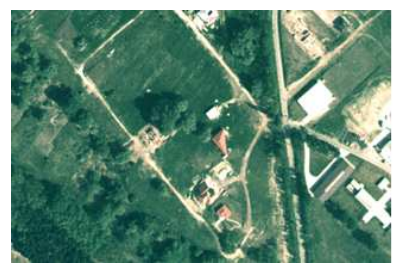

Image $1\left(G_{1}\right)$

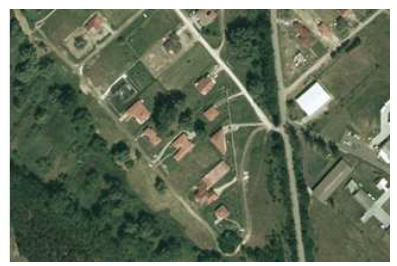

Image $2\left(G_{2}\right)$ 


\section{Task formulation}

- Binary image segmentation problem:

- Classifying each pixel $s$ of the image lattice $S$ as 'change' (below: white) or 'background' (i.e. unchanged, with black)
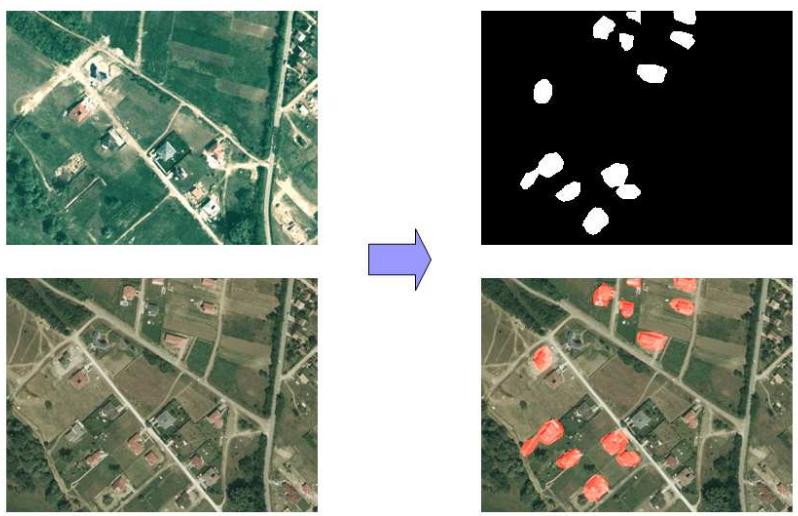


\section{Content}

\section{Introduction}

(2) Feature extraction and integration
- Global intensity statistics
- Local block correlation
- Feature integration

A Mixed Markovian image segmentation model

- Introduction to mixed Markov models

- Proposed model

Experiments 


\section{Feature definition}

- Global statistics of intensity co-occurrences

- Feature vector of pixel $s$ is pair of intensity values of $s$ in the two images: $\bar{g}(s)=\left[g_{1}(s), g_{2}(s)\right]^{T}, g_{1}(s) \in G_{1}, g_{2}(s) \in G_{2}$

- Global statistics in changed/background regions:

a)

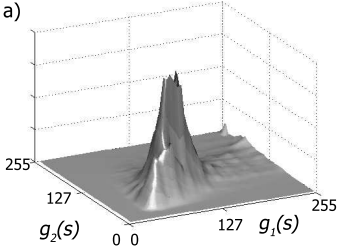

c)

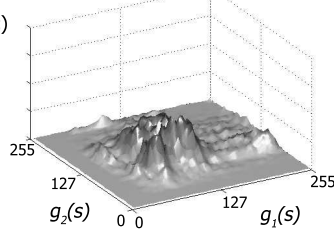

b)

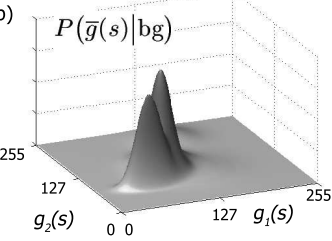

d)

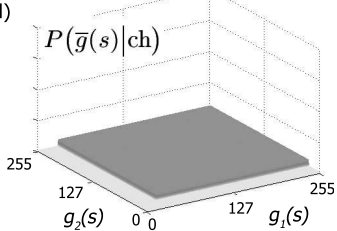




\section{Feature density modeling}

- Multi-Gaussian Intensity-based (MGI) change detection: 'change' class is modeled by a 2-D uniform pdf, while 'background' with a mixture of Gaussians in the $\bar{g}(s)$ feature space

- Class 'background':

$$
P(\bar{g}(s) \mid \mathrm{bg})=\sum_{i=1}^{K} \kappa_{i} \cdot \eta\left(\bar{g}(s), \bar{\mu}_{i}, \Sigma_{i}\right)
$$

- using fixed $K($ e.g. $K=5)$ and EM parameter estimation

- Class 'change':

$$
P(\bar{g}(s) \mid \mathrm{ch})= \begin{cases}\frac{1}{\left(b_{1}-a_{1}\right) \cdot\left(b_{2}-a_{2}\right)}, & \text { if } \bar{g}(s) \in \Gamma \\ 0 & \text { otherwise }\end{cases}
$$

- where $\bar{g}(s) \in \Gamma$ iff $a_{1} \leq g_{1}(s) \leq b_{1}$ and $a_{2} \leq g_{2}(s) \leq b_{2}$ 


\section{Validation of the Intensity Feature}

- Result of the intensity based ML pixel classification

$$
\phi_{g}(s)=\operatorname{argmax}_{\psi \in\{\mathrm{ch}, \mathrm{bg}\}} P(\bar{g}(s) \mid \psi)
$$
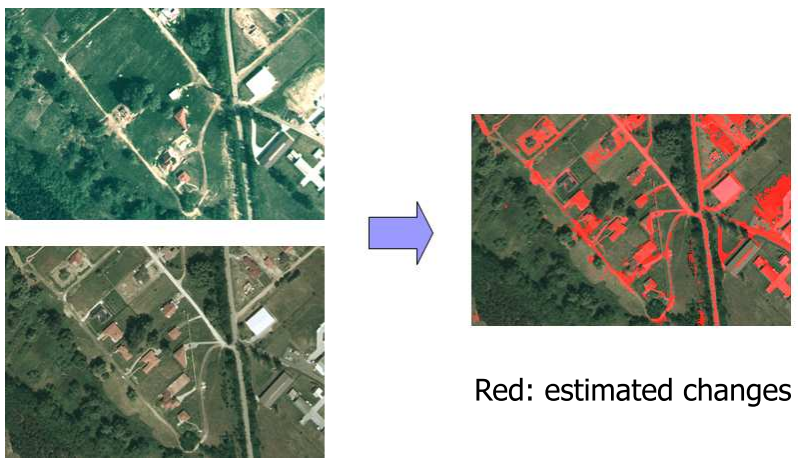

Red: estimated changes

False alarms in textured image regions 


\section{Feature extraction 2}

- Second feature: local block correlation

- $c(s)$ : normalized cross correlation between the $v \times v$ neighborhoods of pixel $s$ in $G_{1}$ resp. $G_{2}$ images (used $v=17$ ).
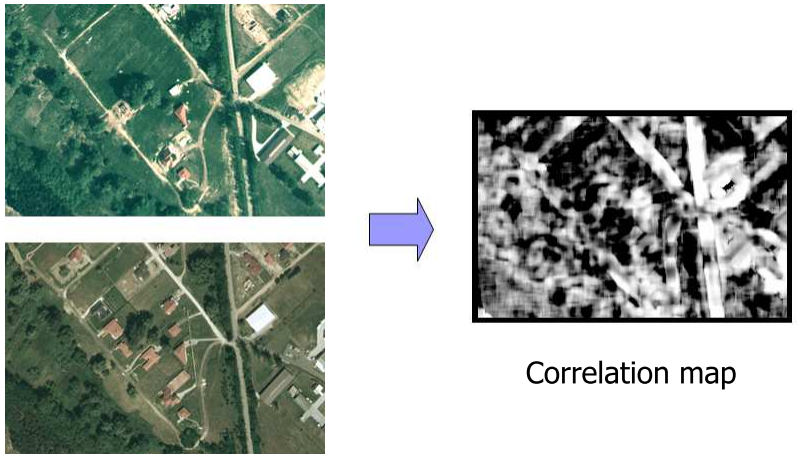

Correlation map 


\section{Feature extraction 2}

- Feature statistics
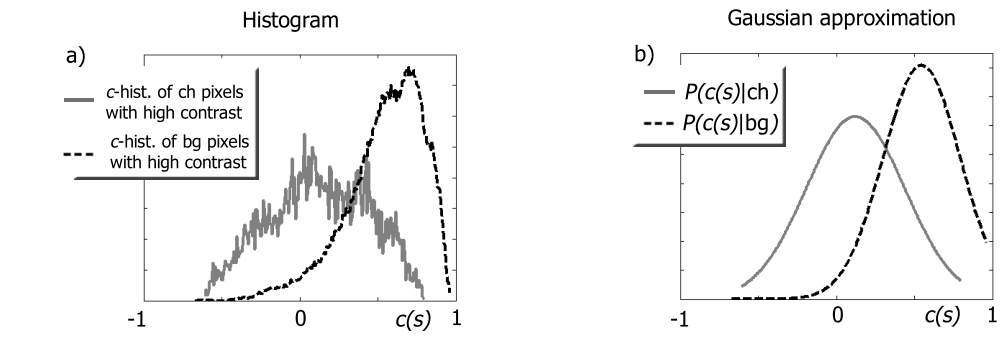

$$
\begin{gathered}
P(c(s) \mid \mathrm{ch})=\eta\left(c(s), \vartheta_{\mathrm{ch}}, \varsigma_{\mathrm{ch}}^{2}\right)=\frac{1}{\sqrt{2 \pi} \varsigma_{\mathrm{ch}}^{2}} \exp \left(-\frac{\left(c(s)-\vartheta_{\mathrm{ch}}\right)^{2}}{2 \varsigma_{\mathrm{ch}}^{2}}\right) \\
P(c(s) \mid \mathrm{bg})=\eta\left(c(s), \vartheta_{\mathrm{bg}}, \varsigma_{\mathrm{bg}}^{2}\right)
\end{gathered}
$$




\section{Feature extraction 2}

- Result of the correlation based ML pixel classification

$$
\phi_{C}(s)=\operatorname{argmax}_{\psi \in\{\mathrm{ch}, \mathrm{bg}\}} P(c(s) \mid \psi)
$$
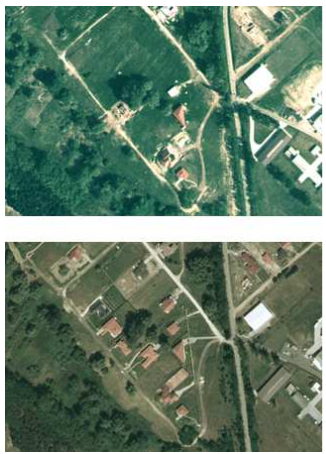

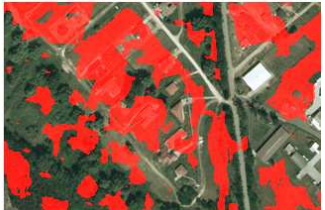

Estimated changes

False alarms in homogenous image regions 


\section{Feature of feature selection}

- Feature selection based on local contrast

- $\nu_{i}(s), i \in\{1,2\}$ : variance of the gray levels over the $v \times v$ neighborhood of $s$ in $G_{i}$

- Joint variance vector: $\bar{\nu}(s)=\left[\nu_{1}(s), \nu_{2}(s)\right]^{T}$

- Local variance (contrast) maps:

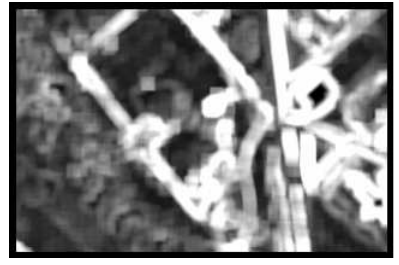

$\nu_{1}($.

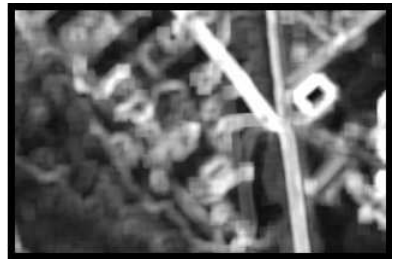

$\nu_{2}($. 


\section{Feature integration}

- Partitioning the pixels of the 'training' image pairs:

$$
S_{\nu_{1}, \nu_{2}}=\left\{\boldsymbol{s} \in \boldsymbol{S} \mid \nu_{1}(\boldsymbol{s}) \approx \nu_{1}, \nu_{2}(\boldsymbol{s}) \approx \nu_{2}\right\}
$$

- Reliability 'histogram' of the intensity map $\phi_{g}$ :

$$
h_{g}\left[\nu_{1}, \nu_{2}\right]=\frac{\text { number of correctly classified pixels in } S_{\nu_{1}, \nu_{2}}}{\text { number of erroneously classified pixels in } S_{\nu_{1}, \nu_{2}}}
$$

- Reliability 'histogram' of the correlation map $\phi_{c}$ :

$$
h_{c}\left[\nu_{1}, \nu_{2}\right]=\frac{\text { number of correctly classified pixels in } S_{\nu_{1}, \nu_{2}}}{\text { number of erroneously classified pixels in } S_{\nu_{1}, \nu_{2}}}
$$




\section{Feature integration}

- Reliability histograms $h_{g}$ and $h_{c}$ with 2-D Gaussian density approximations:
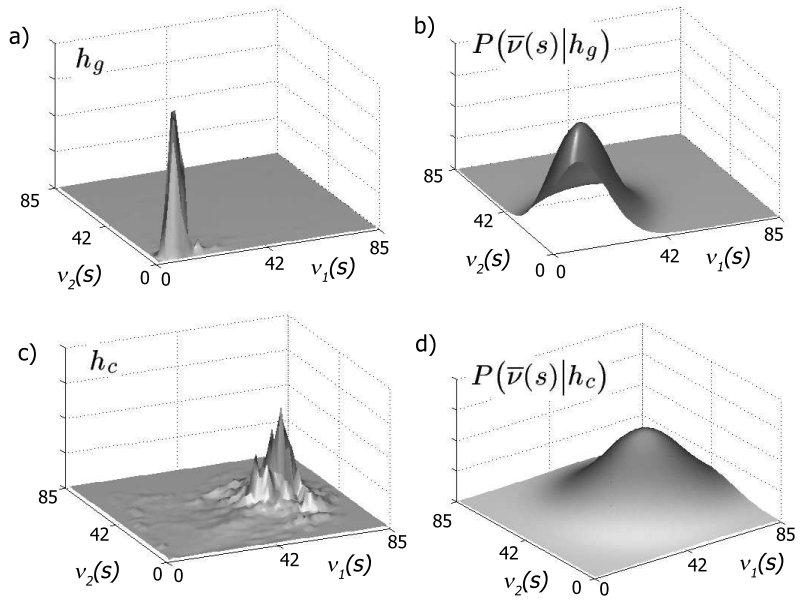


\section{Feature integration}

- Gaussian models for the reliability of the $g / c$ features:

$$
\begin{aligned}
& P\left(\bar{\nu}(s) \mid h_{g}\right)=\eta\left(\bar{\nu}(s), \bar{\mu}_{g}, \bar{\Sigma}_{g}\right) \\
& P\left(\bar{\nu}(s) \mid h_{c}\right)=\eta\left(\bar{\nu}(s), \bar{\mu}_{c}, \bar{\Sigma}_{c}\right)
\end{aligned}
$$

- Contrast-based feature selection-map (red where the correlation feature is estimated as more reliable):

$$
\phi_{\nu}(s)=\operatorname{argmax}_{\chi \in\{g, c\}} P\left(\bar{\nu}(s) \mid h_{\chi}\right) .
$$




\section{Feature integration}

- Initial feature integration rule:

- $\phi_{*}$ : final change mask

$$
\phi_{*}(s)=\left\{\begin{array}{lll}
\phi_{g}(s) & \text { if } \phi_{\nu}(s)=g \\
\phi_{c}(s) & \text { if } \phi_{\nu}(s)=c
\end{array}\right.
$$

- Result of the pixel-by-pixel approach:

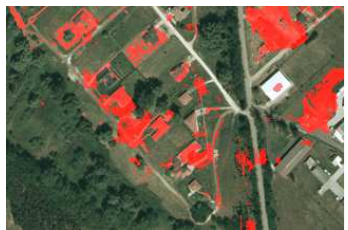

output $\phi_{*}(s)$ map

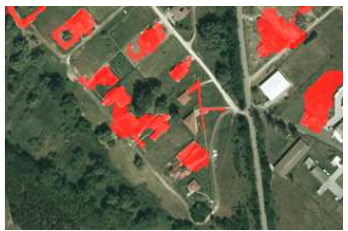

ground truth

- Observation: improved, but still noisy result 


\section{Towards a Robust Segmentation Approach}

- Global labeling optimization over the image instead of pixel-by-pixel segmentation

- pixel level feature descriptions

- interaction constraints between neighbouring pixels

- Conventional Markov Random Field approaches must be extended:

- multi layer model for considering the different label maps

- particular role of the $\bar{\nu}(s)$ feature:

- switching ON and OFF the $\bar{g}(s)$ respectively $c(s)$ features into the integration process

- data dependent dynamic links are needed in the graph

- application of Mixed Markov models 


\section{Content}

\section{Introduction}

Feature extraction and integration

- Global intensity statistics

- Local block correlation

- Feature integration

(3) A Mixed Markovian image segmentation model

- Introduction to mixed Markov models

- Proposed model

Experiments 


\section{Image Segmentation with Conventional MRFs}

- 2-D pixel lattice $\rightarrow$ graph: $S=\{s\}$

- nodes: image points ( $s$ is a pixel)

Lattice $S$

- edges: interactions $\rightarrow$ cliques

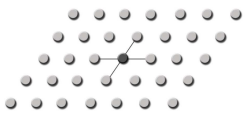

- Goal: generate a $K$-colored segmented image, with segmentation classes: $L=\left\{C_{1}, \ldots, C_{K}\right\}$

- Here: $K=2 ; C_{1}=$ change and $C_{2}=$ background.

- $f_{s}$ : local feature observed at pixel s

- $\omega_{s}$ : label of pixel s which marks its segmentation class

- Segmentation with Markov Random Fields (MRF):

- Pixels' feature-values must agree with the class models specified

by their label:

- Classes are characterized by probability density functions e.g. $P\left(f_{S} \mid \omega_{S}=\right.$ background $)$.

- Segmented image is "smooth": We penalize, if two neighboring pixels have different labels 


\section{Image Segmentation with Conventional MRFs}

- 2-D pixel lattice $\rightarrow$ graph: $S=\{s\}$

- nodes: image points ( $s$ is a pixel)

Lattice $S$

- edges: interactions $\rightarrow$ cliques

- Goal: generate a $K$-colored segmented image, with segmentation classes: $L=\left\{C_{1}, \ldots, C_{K}\right\}$

- Here: $K=2 ; C_{1}=$ change and $C_{2}=$ background.

- $f_{s}$ : local feature observed at pixel $s$

- $\omega_{s}$ : label of pixel s which marks its segmentation class

- Segmentation with Markov Random Fields (MRF):

- Pixels' feature-values must agree with the class models specified

by their label:

- Classes are characterized by probability density functions e.g.

$P\left(f_{s} \mid \omega_{s}=\right.$ background $)$

- Segmented image is "smooth": We penalize, if two neighboring pixels have different labels 


\section{Image Segmentation with Conventional MRFs}

- 2-D pixel lattice $\rightarrow$ graph: $S=\{s\}$

- nodes: image points ( $s$ is a pixel)

Lattice $S$

- edges: interactions $\rightarrow$ cliques

- Goal: generate a $K$-colored segmented image, with segmentation classes: $L=\left\{C_{1}, \ldots, C_{K}\right\}$

- Here: $K=2 ; C_{1}=$ change and $C_{2}=$ background.

- $f_{s}$ : local feature observed at pixel $s$

- $\omega_{s}$ : label of pixel $s$ which marks its segmentation class

- Segmentation with Markov Random Fields (MRF):

- Pixels' feature-values must agree with the class models specified by their label:

- Classes are characterized by probability density functions e.g. $P\left(f_{s} \mid \omega_{s}=\right.$ background $)$.

- Segmented image is "smooth": We penalize, if two neighboring pixels have different labels 


\section{Image Segmentation with Conventional MRFs}

- Global labeling: $\left.\underline{\omega}=\left\{\omega_{S} \mid s \in S\right\}\right\}$

- Observation process: $\mathcal{F}=\left\{f_{s} \mid s \in S\right\}$

- MAP estimation of the optimal global labeling:

$$
\underline{\widehat{\omega}}=\operatorname{argmax}_{\underline{\omega} \in \Omega} P(\underline{\omega} \mid \mathcal{F})
$$

where $\Omega$ denotes the set of all the possible global labelings.

- (Hammersley-Clifford theorem): $P(\underline{\omega} \mid \mathcal{F})$ can be factorized into individual terms whose domains are the cliques of the graph.

$$
P(\underline{\omega} \mid \mathcal{F}) \propto \underbrace{\prod_{s \in S} P\left(f_{S} \mid \omega_{S}\right)}_{P(\mathcal{F} \mid \underline{\omega})} \cdot \underbrace{\frac{1}{Z} \exp \left(-\sum_{C \in \mathcal{C}} V_{C}(\underline{\omega})\right)}_{P(\underline{\omega})}
$$

- where $C$ is an arbitrary clique and $V_{C}$ is the potential of $C$. 


\section{Step forward to Mixed Markov models}

- In MRFs two nodes directly interact if and only if they are connected by a (static) edge

- In Mixed models the connections can also be data dependent

- Two types of nodes:

- regular nodes: same role as nodes of MRF's

- address nodes: their 'labels' are pointers to regular nodes

- Regular nodes A and B may interact iff they are connected by (i) a (static) edge OR (ii) a chain of a static edge and a dynamic address pointer
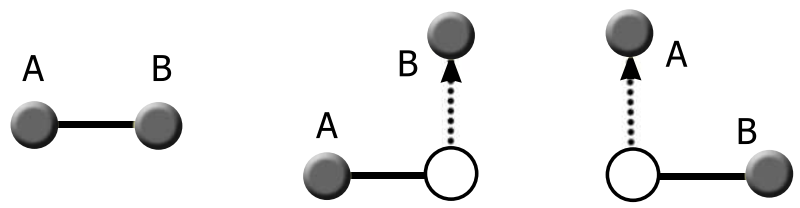

Three cases when $A$ and $B$ regular nodes may interact (address nodes are marked by white circles, edges by lines, pointers by dotted arrows) 


\section{Probability modeling in Mixed Markov models}

- A priory probability of a global labeling:

$$
P(\underline{\omega})=\frac{1}{Z} \exp \left(-\sum_{C \in \mathcal{C}} V_{C}\left(\omega_{C}, \omega_{C}^{\mathcal{A}}\right)\right)
$$

- where $C$ is a clique and $\omega_{C}$ is the set of labels inside $C$ :

$$
\omega_{C}=\{\omega(q) \mid q \in C\}
$$

- while $\omega_{C}^{\mathcal{A}}$ is the set of node labels pointed by the address nodes of clique $C$ :

$$
\omega_{C}^{\mathcal{A}}=\{\tilde{\omega}(a) \mid a \in \mathcal{A} \cap \mathcal{C}, \omega(a) \neq \text { nil }\}
$$

- $\mathcal{A}$ is the set of address nodes and $\tilde{\omega}(a)=\omega(\omega(a))$ for $a \in \mathcal{A}$ 


\section{4-layer Mixed Markov model for Change Detection}

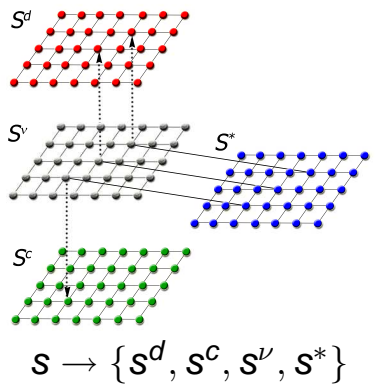

- Regular layers

- $S^{g}, S^{c}$ : change masks based on the $\bar{g}(s)$ resp. $c(s)$ features

- $S^{*}$ : combined layer - output change mask

- Address layer

- $S^{\nu}$ : switch layer providing configurable, data-driven inter-layer connections

- Node labels: $\omega\left(s^{i}\right): i \in\{d, c, \nu, *\}, s \in S$ 


\section{4-layer Mixed Markov model for Change Detection}

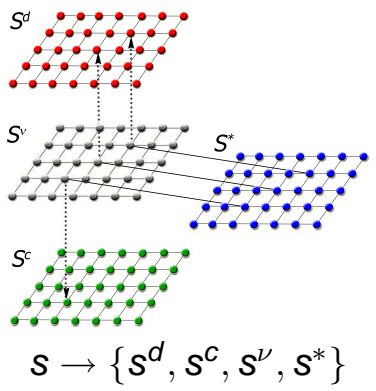

- Regular layers

- $S^{g}, S^{c}$ : change masks based on the $\bar{g}(s)$ resp. $c(s)$ features

- $S^{*}$ : combined layer - output change mask

- Address layer

- $S^{\nu}$ : switch layer providing configurable, data-driven inter-layer connections

- Node labels: $\omega\left(s^{i}\right): i \in\{d, c, \nu, *\}, s \in S$

- Cliques and clique potentials:

- Singletons: data - label consistency

- Intra-layer connections: smooth label maps $V_{C_{2}}$

- Inter-layer interactions: label fusion $V_{C_{3}}$ 


\section{Singleton terms}

- Assuming conditional independent observations, let be:

$$
P(\mathcal{F} \mid \Omega)=\prod_{s \in S} P\left(\bar{g}(s) \mid \omega\left(s^{g}\right)\right) \cdot P\left(c(s) \mid \omega\left(s^{c}\right)\right) \cdot P\left(\bar{\nu}(s) \mid \omega\left(s^{\nu}\right)\right)
$$

where we use previously defined densities for the $S^{g}$ and $S^{c}$ layers:

$$
\begin{gathered}
P\left(\bar{g}(s) \mid \omega\left(s^{g}\right)=\mathrm{bg}\right)=\sum_{i=1}^{K} \kappa_{i} \cdot \eta\left(\bar{g}(s), \bar{\mu}_{i}, \Sigma_{i}\right) \\
P\left(\bar{g}(s) \mid \omega\left(s^{g}\right)=\mathrm{ch}\right)=1 /\left[\left(b_{1}-a_{1}\right) \cdot\left(b_{2}-a_{2}\right)\right] \\
P\left(c(s) \mid \omega\left(s^{c}\right)=\psi\right)=\eta\left(c(s), \vartheta_{\psi}, \varsigma_{\psi}^{2}\right), \psi \in\{\mathrm{ch}, \mathrm{bg}\}
\end{gathered}
$$

Singletons of $S^{\nu}$ will be later given. 


\section{Intra-layer Doubleton Potentials}

- Doubleton cliques: smoothing priors of the segmentation within each layer.

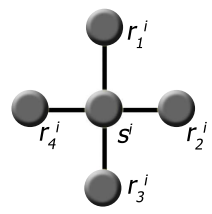

- The potential of an intra-layer clique $C_{2}=\left\{s^{i}, r^{i}\right\} \in \mathcal{C}_{2}$, $i \in\{g, c, *, \nu\}$ :

$$
V_{C_{2}}= \begin{cases}-\delta^{i} & \text { if } \omega\left(s^{i}\right)=\omega\left(r^{i}\right) \\ +\delta^{i} & \text { if } \omega\left(s^{i}\right) \neq \omega\left(r^{i}\right)\end{cases}
$$

for a constant $\delta^{i}>0$. 


\section{Inter-layer interactions}

- Inter-layer cliques: $\omega\left(s^{*}\right)$ should mostly be equal either to $\omega\left(s^{g}\right)$ or to $\omega\left(s^{c}\right)$, depending on the 'vote' of the $\nu(s)$ feature.

- Edge between $s^{*}$ and $s^{\nu}$

- Address node $s^{\nu}$ should point either to $s^{g}$ or to $s^{c}$ :

$$
\forall s \in S: \omega\left(s^{\nu}\right) \in\left\{s^{g}, s^{c}\right\}
$$

a)

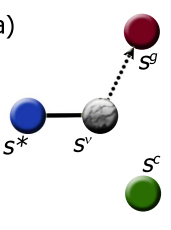

b)

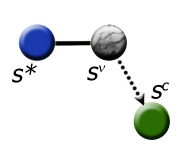

- The directions of the address pointers are influenced by the singletons of $S^{\nu}$ :

$$
P\left(\bar{\nu}(s) \mid \omega\left(s^{\nu}\right)=s^{\chi}\right)=P\left(\bar{\nu}(s) \mid h_{\chi}\right), \quad \chi \in\{g, c\}
$$




\section{Inter-layer interactions}

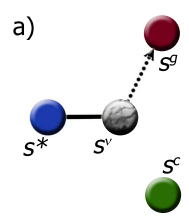

b)

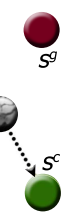

- The potential function of the inter-layer clique $C_{3}=\left\{s^{*}, s^{\nu}\right\}$ :

$$
V_{C_{3}}\left(\omega\left(s^{*}\right), \tilde{\omega}\left(s^{\nu}\right)\right)= \begin{cases}-\rho & \text { if } \omega\left(s^{*}\right)=\tilde{\omega}\left(s^{\nu}\right) \\ +\rho & \text { otherwise }\end{cases}
$$

where $\rho>0$, and $\tilde{\omega}\left(s^{\nu}\right)=\omega\left(\omega\left(s^{\nu}\right)\right)$. 


\section{Labeling optimization}

- MAP estimation of the optimal global labeling $\underline{\hat{\hat{\omega}}}$ :

$$
\begin{gathered}
\underline{\widehat{\omega}}=\arg \min _{\underline{\omega} \in \Omega}\left\{\sum_{s \in S}-\log P\left(\bar{g}(s) \mid \omega\left(s^{g}\right)\right)+\right. \\
+\sum_{s \in S}-\log P\left(c(s) \mid \omega\left(s^{c}\right)\right)+\sum_{s \in S}-\log P\left(\bar{\nu}(s) \mid \omega\left(s^{\nu}\right)\right)+ \\
\left.+\sum_{i ;\{s, r\} \in \mathcal{C}_{2}} V_{C_{2}}\left(\omega\left(s^{i}\right), \omega\left(r^{i}\right)\right)+\sum_{s \in S} V_{C_{3}}\left(\omega\left(s^{*}\right), \tilde{\omega}\left(s^{\nu}\right)\right)\right\}
\end{gathered}
$$

- Optimization by simulated annealing (Modified Metropolis algorithm)

- Output: labeling of the $S^{*}$ layer. 


\section{Content}

\section{(1) Introduction}

(2) Feature extraction and integration
- Global intensity statistics
- Local block correlation
- Feature integration

\section{A Mixed Markovian image segmentation model \\ - Introduction to mixed Markov models \\ - Proposed model}

\section{(4) Experiments}




\section{Test datasets and reference methods}

- Database: three sets of optical aerial image pairs provided by the Hungarian Institute of Geodesy Cartography \& Remote Sensing (FÖMI) and Google Earth.

- Data set SzADA: images by FÖMI from 2000 resp. 2005. Seven also manually evaluated - photo pairs, covering in aggregate $9.5 \mathrm{~km}^{2}$ area at $1.5 \mathrm{~m} / \mathrm{pixel}$ resolution.

- Data set TISZADOB: five photo pairs from 2000 resp. 2007 (6.8km²) with similar size and quality parameters to SzADA.

- Test pair ARCHIVE, an aerial image taken by FÖMI in 1984 and a corresponding Google Earth photo from around 2007.

- Manually generated ground truth masks

- Metrics: number of false and missed alarms

- 4 reference methods: PCA, Hopfield, MLP, Parzen 


\section{Ground truth generation}

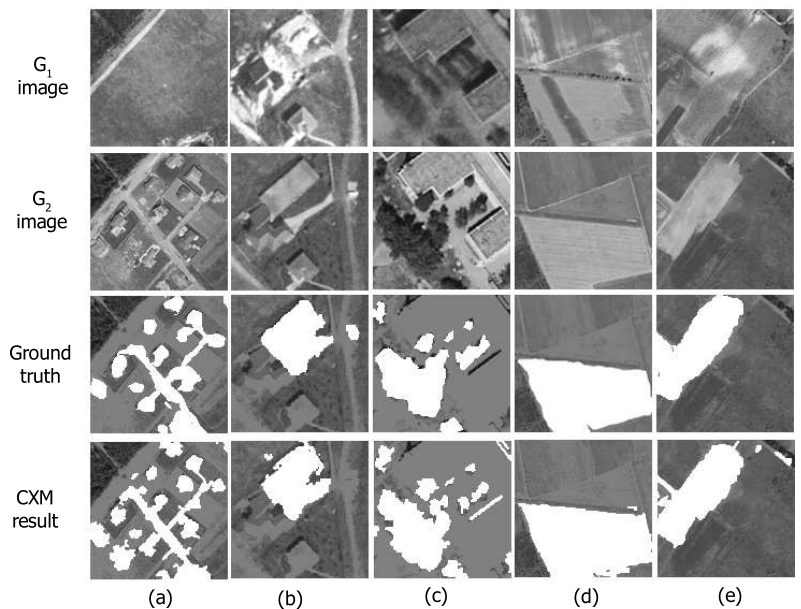

Change prototypes considered for ground truth generation (a) new built-up regions (b) building operations (c) planting of trees (d) fresh plough-land (e) groundwork before building over 


\section{Quantitative comparison}
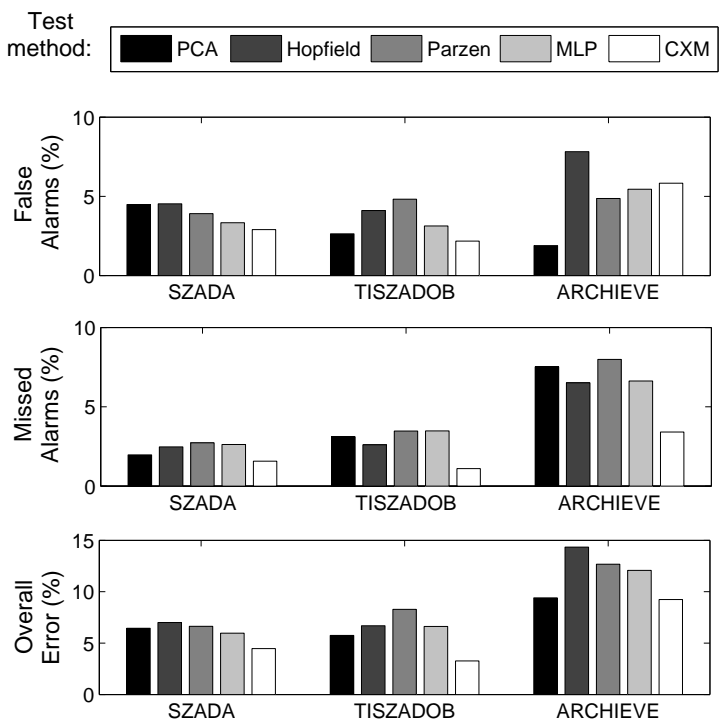


\section{Qualitative comparison}

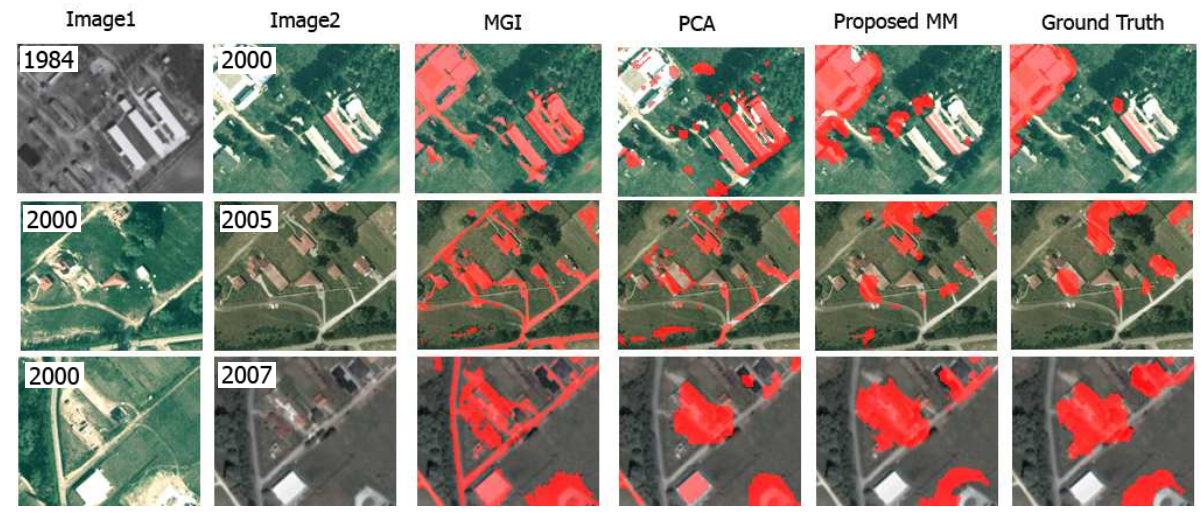




\section{Qualitative comparison}

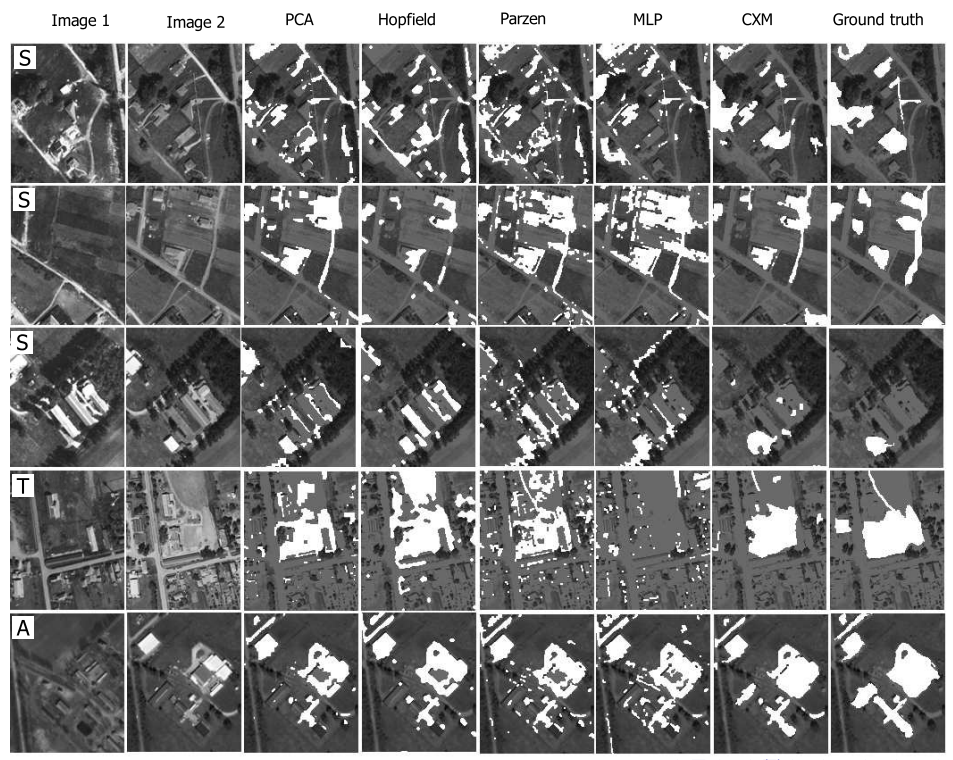




\section{References}

- Cs. Benedek and T. Szirányi: "Change Detection in Optical Aerial Images by a Multi-Layer Conditional Mixed Markov Model", submitted to IEEE Transacions of Geosciences and Remote Sensing, 2008, before $2^{\text {nd }}$ review round

- Cs. Benedek and T. Szirányi: "A Mixed Markov Model for Change Detection in Aerial Photos with Large Time Differences", International Conference on Pattern Recognition (ICPR), Tampa, Florida, USA, December 8-11, 2008 


\section{Acknowledgement and contacts}

- The authors would like to thank

- Josiane Zerubia from INRIA for her kind advices regarding the proposed model

- the MUSCLE Shape Modeling E-Team for financial support of this work

- Prof. Anuj Srivastava for inviting me to the Florida State University

- the Associated team Shapes (INRIA, FSU) for supporting my visit to FSU

- Contact me: Csaba Benedek

- Url: http://web.eee.sztaki.hu/ bcsaba/

- E-mail: cbenedekesophia.inria.fr, bcsabalsztaki.hu 